\title{
Calidad de vida relacionado con la salud bucal en adultos de la ciudad de Quito - Ecuador
}

Qualidade de vida relacionada com a saúde oral em adultos na cidade de Quito - Equador

Quality of life related to oral health in adults in the city of Quito - Ecuador

Tonny Fabián Muñoz Torres tfmunozt45@est.ucacue.edu.ec Odontólogo General. Universidad Católica de Cuenca- Cuenca, Ecuador https://orcid.org/0000-0001-7764-200X

\section{Celia María Pulgarín Fernández} celia.pulgarin@ucacue.edu.ec Médico Intensivista, Hospital José Carrasco Arteaga, Cuenca, Ecuador, Docente de la Universidad Católica de Cuenca, Cuenca, Ecuador https://orcid.org/0000-0002-5653-9078

\section{Miriam Fernanda Ortega López} mfortegal38@hotmail.com Especialista en Docencia Clínica Odontológica, Universidad del Zulia, Maracaibo, Venezuela. Especialista en Odontopediatría, Universidad UTE, Quito- Ecuador. https://orcid.org/0000-0001-7736-3617

\section{Ronald Roossevelt Ramos Montiel}

ronald_mtz@hotmail.com rramosm@ucacue.edu.ec

Especialista en Ortodoncia, Especialista en Docencia Clínica Odontológica, Doctor en Ciencias Odontológicas. Coordinador de la Especialización en Ortodoncia y Docente de Grado de la Unidad Académica de Salud y Bienestar de la Universidad Católica de Cuenca. Cuenca-Ecuador. Miembro de la Sociedad Ecuatoriana de Ortodoncia y Ortopedia, Asociación Latinoamericana de Ortodoncia y Federación Mundial de Ortodoncistas. https://orcid.org/0000-0002-8066-5365

\section{RESUMEN}

Antecedentes: La salud bucal es un componente esencial de la salud general y se ha reconocido que es fundamental para la determinación de la CVRS por lo que la calidad de vida relacionada con la salud bucal (CVRSB) también es un constructo multidimensional que expresa el grado de percepción sobre la comodidad, satisfacción o insatisfacción de una persona en su vida diaria respecto a su salud bucal. Objetivo: Determinar la calidad de vida relacionada con la salud bucal en adultos de la ciudad de Quito-Ecuador 2021. Materiales y métodos: estudio descriptivo; trasversal, se utilizó el muestreo no probabilístico de redes o bola de nieve. El proceso consistió en localizar a personas residentes en la Ciudad de Quito que tengan 18 años en adelante a través de las plataformas virtuales correo electrónico, WhatsApp y Facebook. La muestra quedo conformada por 403 participantes. El cuestionario recogió datos sociodemográficos y los ítems de OHIP-14. Resultados: El 65\% de los encuestados reportó tener ingresos inferiores a 400\$ mensuales y el 85\% no contaba con un seguro odontológico. Respecto a la CVRSB se encontró que solo en la dimensión malestar psicológico un porcentaje importante de los individuos encuestados auto-informaron una percepción negativa de su CVRSB (32\% de la sumatoria a menudo, casi siempre y siempre). Conclusiones: Respecto a la CVRSB, un porcentaje importante de los individuos encuestados auto-informaron una percepción negativa en la dimensión malestar psicológico. No así en las demás dimensiones. Los resultados de las mediciones de del OHIP-14 pudieron estar influenciados por el bajo nivel de ingreso de los individuos que los ubica como población vulnerable y ello pudo intervenir en la percepción de su CVRSB ya que al enfrentar necesidades más urgentes para sus condiciones materiales de vida pudieran estar restando importancia al impacto de sus afecciones bucales.

Palabras clave: calidad de vida relacionada con salud bucal, adultos.

\section{RESUMO}

Antecedentes: A saúde oral é uma componente essencial da saúde geral e tem sido reconhecida como central para a determinação da HRQoL, pelo que a qualidade de vida relacionada com a saúde oral (OHRQoL) é também uma construção multidimensional que expressa o grau de conforto, satisfação ou insatisfação percebido por uma pessoa na 
sua vida quotidiana no que diz respeito à sua saúde oral. Objectivo: Determinar a qualidade de vida relacionada com a saúde oral em adultos na cidade de Quito-Equador 2021.

Materiais e métodos: estudo descritivo; foi utilizada uma amostragem transversal, não-probabilística de redes ou bola de neve. O processo consistia em localizar pessoas que vivem na cidade de Quito com 18 anos de idade ou mais através das plataformas virtuais e-mail, WhatsApp e Facebook. A amostra consistiu em 403 participantes. O questionário recolheu dados sociodemográficos e itens OHIP-14. Resultados: 65\% dos inquiridos declararam ter um rendimento inferior a 400 dólares por mês e $85 \%$ não tinham seguro dentário. Em relação ao CVRSB, verificou-se que apenas na dimensão de angústia psicológica uma percentagem significativa dos inquiridos se auto-relatava uma percepção negativa do seu CVRSB (32\% da soma de muitas vezes, quase sempre e sempre). Conclusões: Relativamente ao CVRSB, uma percentagem significativa dos inquiridos auto-relatou uma percepção negativa na dimensão do sofrimento psicológico. Este não foi o caso para as outras dimensões. Os resultados das medições do OHIP-14 poderiam ser influenciados pelo baixo nível de rendimento dos indivíduos, o que os coloca como uma população vulnerável, e isto poderia intervir na percepção do seu CVRSB, uma vez que enfrentam necessidades mais urgentes para as suas condições materiais de vida e poderiam estar a minimizar o impacto das suas condições orais.

Palavras-chave: qualidade de vida relacionada com a saúde oral, adultos.

\begin{abstract}
Introduction: Oral health is an essential component of general health and it has been recognized that it is fundamental for the determination of HRQoL, therefore the quality of life related to oral health (HRQL) is also a multidimensional construct that expresses the degree of perception about the comfort, satisfaction or dissatisfaction of a person in their daily life regarding their oral health. Objective: To determine the quality of life related to oral health in adults in the city of Quito-Ecuador 2021. Materials and methods: descriptive study; cross-sectional, non-probabilistic network or snowball sampling was used. The process consisted of locating people residing in the City of Quito who are 18 years old and over through the virtual platforms email, WhatsApp and Facebook. The sample was made up of 403 participants. The questionnaire collected sociodemographic data and the OHIP-14 items. Results: $65 \%$ of those surveyed reported having an income of less than $\$ 400$ per month and $85 \%$ did not have dental insurance. Regarding the HRQL, it was found that only in the psychological distress dimension a significant percentage of the individuals surveyed self-reported a negative perception of their HRQL (32\% of the sum often, almost always and always). Conclusions: Regarding the HRQL, a significant percentage of the surveyed individuals self-reported a negative perception in the psychological distress dimension. Not so in the other dimensions. The results of the OHIP-14 measurements could be influenced by the low level of income of the individuals that places them as a vulnerable population and this could intervene in the perception of their HRQL since when facing more urgent needs for their material conditions of life could be downplaying the impact of oral conditions.
\end{abstract}

Key words: quality of life related to oral health, adults.

\title{
1. INTRODUCCIÓN
}

La calidad de vida relacionada con salud (CVRS) se ha definido como el nivel percibido de bienestar, derivado de la evaluación que realiza cada persona sobre elementos objetivos y subjetivos en distintas dimensiones y etapas de su vida ${ }^{1}$, también depende del contexto cultural donde se desenvuelve el individuo la edad, el género el nivel de educación y el estatus socioeconómico. ${ }^{2}$ Schwartzmann $^{3}$ sostiene que la CVRS es un concepto multidimensional y para medirla o establecerla se debe incluir la percepción de los individuos respecto a su bienestar físico, emocional y social; se debe partir entonces de reconocer que es un reflejo de la manera positiva o negativa en la que el sujeto percibe su estado de salud ${ }^{4}$.

La Organización Mundial de la Salud (OMS), la ha definido como "Percepción del individuo sobre su posición en la vida, en el contexto de la cultura y sistema de valores en el cual él vive, y en relación con sus objetivos, expectativas, estándares e intereses" y ha establecido un instrumento para evaluar la CVRS en el que se intenta trascender la medición de la salud solo mediante indicadores de mortalidad y morbilidad; y que incluye aquellos aspectos que pueden afectar la salud de los individuos en el diario desenvolvimiento de su vida. 
Ese instrumento es el WHOQOL-100 (por sus siglas en Ingles) que indaga como el paciente afronta su enfermedad y su percepción sobre el bienestar o malestar de acuerdo con las experiencias vividas. Esta percepción es el resultado de la intervención de una serie de factores entre ellos el nivel socioeconómico ${ }^{6,7}$ las variables socio-demográficas y las condiciones de salud ${ }^{8}$ entre otras.

La salud bucal es un componente esencial de la salud general y se ha reconocido que es fundamental para la determinación de la $\mathrm{CVRS}^{8,9}$ por lo que la calidad de vida relacionada con la salud bucal (CVRSB) también es un constructo multidimensional que expresa el grado de percepción sobre la comodidad, satisfacción o insatisfacción de una persona en su vida diaria respecto a su salud bucal. ${ }^{10}$

Investigaciones previas han evaluado el impacto de las enfermedades bucodentales en la CVRSB, en niños y adolescentes ${ }^{11,12,13}$ adultos jovenes ${ }^{8,14}$ y adultos mayores ${ }^{15,16,17}$. La revisión sistemática efectuada por $\mathrm{Haag}^{18}$ reportó que diez estudios de los evaluados encontraron impacto negativo de la pérdida dental en la CVRSB y cuatro informaron que la enfermedad periodontal afectó la CVRSB.

Así mismo una revisión sistemática reciente de estudios realizados en América Latina y el Caribe para evaluar el Impacto de las enfermedades bucodentales en la calidad de vida relacionada con la salud bucal ${ }^{10}$ encontró que la mayoría de los estudios seleccionados reportaron impacto negativo de las enfermedades bucales en la CVRSB y que a mayor gravedad de la enfermedad bucal mayor era el impacto en la CVRSB sin embargo, sus autores también reportaron que la representatividad de este tipo de estudios en los países de ALC fue muy baja a excepción de Brasil, lo que demuestra la falta de investigación en el área de la salud bucal en esta región relacionado con esta temática.

Estas razones justifican la realización de esta investigación que se planteó como objetivo determinar la calidad de vida relacionada con la salud bucal (CVRSB) en los adultos de la ciudad de Quito-Ecuador 2021.

\section{MATERIALES Y MÉTODOS}

Este fue un estudio descriptivo; trasversal, se utilizó el muestreo no probabilístico denominados redes a bola de nieve ${ }^{19}$ el cual se basa en referencias de sujetos iniciales para generar sujetos adicionales. El proceso consistió en localizar a personas residentes en la Ciudad de Quito en edades comprendidas entre 18 años en adelante, a través de las plataformas virtuales correo electrónico, WhatsApp y Facebook para responder un cuestionario al cual se tenía acceso por medio de un enlace a Google Form (https://www.google.com/forms/about) y se les solicitó a su vez compartirlo con otras personas o comunidades virtuales que cumplan con el rango de edad.

El cuestionario estuvo disponible desde el 30 de mayo al 25 de junio de 2021. Se recibió un total de 403 encuestas y se estableció como criterios de exclusión, aquellos participantes que enviaron las mismas en fechas posteriores a las establecidas.

El cuestionario estuvo estructurado en tres aspectos; en el primero se explicó el propósito del estudio y se requirió el consentimiento para la participación; en el segundo se solicitaron datos socio-demográficos; edad, sexo, nivel de ingresos, sitio donde acudían para recibir atención odontológica y tenencia o no de seguro de atención dental. El tercer apartado lo constituyeron los ítems establecidos en el instrumento del Perfil de Impacto en Salud Oral conocido como OHIP-14 por sus siglas en inglés; en su versión español ${ }^{20}$ que evalúa la auto percepción de los individuos respecto a su CVRSB.

\section{ANÁLISIS ESTADÍSTICO}


Se utilizaron medidas de dispersión (media, límites superior e inferior y desviación estándar) para el procesamiento de datos, mediante el paquete estadístico IBM/SPSS Versión 26.

\section{ASPECTOS ÉTICOS}

El presente estudio fue aprobado por el comité de Bioética de la Universidad Católica de Cuenca, Ecuador y se solicitó vía web el consentimiento de todos los participantes que decidieron responder el cuestionario en el cual además de explicar los propósitos del estudio se garantizó el anonimato de los participantes y la confidencialidad de los datos proporcionados. La aprobación se encuentra disponible en el Anexo 1.

\section{RESULTADOS}

Las características sociodemográficas de los individuos del estudio se observan en la tabla 1. La muestra estuvo constituida por 403 personas de las cuales $58,8 \%(n=237)$ eran mujeres y $41,2 \%$ $(\mathrm{n}=166)$ hombres. El 84,5\% $(\mathrm{n}=341)$ pertenecían al grupo de edad de 18 a 44 años, en el rango de edad de 45 a 64 años se encuentra el 12.7\% ( $n=51)$. Los adultos mayores representaron el $2.8 \%$ (11) de la muestra. El 65\% ( $\mathrm{n}=262)$ reportó tener ingresos inferiores al salario básico de $400 \$$ mensuales.

Los servicios de atención odontológica que utilizaban los participantes encuestados y su financiamiento mediante seguros dentales se muestran en la tabla 2. Al presentar un problema de salud bucal un 58\% $(\mathrm{n}=234)$ indico que preferían utilizar los servicios de los consultorios privados particulares, por otro lado, un $85 \%(n=343)$ de los encuestados no contaba con un seguro odontológico.

Respecto a la CVRSB tabla 3, se pudo observar los individuos encuestados auto informaron una percepción de su CVRSB negativa en la dimensión malestar psicológico señalaron que a menudo les preocupaban los problemas con su boca y a menudo también se sentían estresados por esos mismos problemas.

Tabla 1. Características Socio-demográficas de los participantes del estudio

\begin{tabular}{lcc}
\hline Características & $\mathrm{n}=403$ & $\%=100$ \\
\hline $\begin{array}{l}\text { Sexo } \\
\text { Femenino }\end{array}$ & 237 & 58.8 \\
Masculino & 166 & 41.2 \\
Grupos de Edades & & \\
18 a 44 años & 341 & 84.5 \\
45 a 64 años & 51 & 12.7 \\
65 a más años & 11 & 2.8 \\
Nivel de Ingreso & & \\
$<$ de 400\$ & 262 & 65.0 \\
$\geq 400$ a más & 141 & 35.0 \\
\hline
\end{tabular}


Tabla 2. Servicios de atención odontológica y seguro dental de los participantes del estudio

\begin{tabular}{lcc}
\hline $\begin{array}{l}\text { Cuando tiene un problema de } \\
\text { salud bucal, ¿a cuál } \\
\text { establecimiento de salud asiste? }\end{array}$ & $\mathrm{n}=403$ & $\%=100$ \\
\hline $\begin{array}{l}\text { Consultorios privados } \\
\text { (particulares) }\end{array}$ & 234 & 58 \\
IESS & 72 & \\
Ministerio de Salud Pública & 86 & 21 \\
No asisto a ninguno & 11 & 03 \\
& & \\
¿Tiene usted seguro dental? & 343 & 85 \\
$\quad$ No & 60 & 15 \\
$\quad \mathrm{Si}$ & & \\
\hline
\end{tabular}

Tabla 3. Calidad de vida relacionada con la salud bucal OHIP.

\begin{tabular}{|c|c|c|c|c|c|c|c|c|c|c|c|c|}
\hline \multirow{2}{*}{ Preguntas } & \multicolumn{2}{|c|}{ Nunca } & \multicolumn{2}{|c|}{ Casi nunca } & \multicolumn{2}{|c|}{ A menudo } & \multicolumn{2}{|c|}{ Casi siempre } & \multicolumn{2}{|c|}{ Siempre } & \multicolumn{2}{|c|}{ Total } \\
\hline & $\mathbf{n}$ & $\%$ & $\mathbf{n}$ & $\%$ & $\mathbf{n}$ & $\%$ & $\mathbf{n}$ & $\%$ & $\mathbf{n}$ & $\%$ & $\mathbf{n}$ & $\%$ \\
\hline \multicolumn{13}{|l|}{ Limitación Funcional } \\
\hline $\begin{array}{l}\text { 1. ¿Ha tenido dificultad para pronunciar } \\
\text { palabras? }\end{array}$ & 268 & 66.5 & 98 & 24.3 & 27.0 & 6.7 & 7 & 1.7 & 3 & 0.7 & 403 & 100 \\
\hline $\begin{array}{l}\text { 2. ¿El sabor de sus alimentos ha } \\
\text { empeorado? } \\
\text { Dolor Físico }\end{array}$ & 291 & 72 & 79 & 20 & 25 & 6 & 6 & 2 & 2 & 1 & 403 & 100 \\
\hline 3. ¿Ha presentado molestias al comer? & 202 & 50 & 151 & 38 & 35 & 9 & 11 & 3 & 4 & 1 & 403 & 100 \\
\hline 4. ¿Ha sentido dolor en su boca? & 213 & 53 & 138 & 34 & 36 & 9 & 15 & 4 & 1 & 0 & 403 & 100 \\
\hline \multicolumn{13}{|l|}{ Malestar Psicológico } \\
\hline $\begin{array}{l}\text { 5. ¿Le preocupan los problemas con su } \\
\text { boca? }\end{array}$ & 165 & 41 & 110 & 27 & 59 & 15 & 29 & 7 & 40 & 10 & 403 & 100 \\
\hline 6. ¿Se ha sentido estresado? & 192 & 47.6 & 138 & 34.2 & 49.0 & 12.2 & 11 & 2.7 & 13 & 3.2 & 403 & 100 \\
\hline \multicolumn{13}{|l|}{ Discapacidad Fisica } \\
\hline $\begin{array}{l}\text { 7. ¿Ha tenido que cambiar sus alimentos? } \\
\text { ( Comer cosas blandas) }\end{array}$ & 237 & 58.8 & 113 & 28.0 & 35.0 & 8.7 & 15 & 3.7 & 3 & 0.7 & 403 & 100 \\
\hline $\begin{array}{l}\text { 8. ¿Ha tenido que interrumpir sus } \\
\text { alimentos? (Hacer una pausa para comer) }\end{array}$ & 239 & 59.3 & 119 & 29.5 & 33.0 & 8.2 & 8 & 2.0 & 4 & 1.0 & 403 & 100 \\
\hline \multicolumn{13}{|l|}{ Discapacidad Psicológica } \\
\hline $\begin{array}{l}\text { 9. ¿Ha tenido dificultad para descansar? } \\
\text { (Dormir) }\end{array}$ & 261 & 64.8 & 104 & 25.8 & 28 & 6.9 & 8 & 2.0 & 2 & 0.5 & 403 & 100 \\
\hline $\begin{array}{l}\text { 10. ¿Se ha sentido avergonzado por } \\
\text { problemas con su boca? }\end{array}$ & 214 & 53.1 & 124 & 30.8 & 48 & 11.9 & 13 & 3.2 & 4 & 1.0 & 403 & 100 \\
\hline \multicolumn{13}{|l|}{ Discapacidad social } \\
\hline $\begin{array}{l}\text { 11. ¿Ha estado irritable debido a problemas } \\
\text { con su boca? }\end{array}$ & 227 & 56.3 & 124 & 30.8 & 35 & 8.7 & 10 & 2.5 & 7 & 1.7 & 403 & 100 \\
\hline $\begin{array}{l}\text { 12. ¿Ha tenido un poco de dificultad para } \\
\text { realizar sus actividades diarias? }\end{array}$ & 273 & 67.7 & 101 & 25.1 & 20 & 5 & 8 & 2.0 & 1 & 0.2 & 403 & 100 \\
\hline \multicolumn{13}{|l|}{ Minusvalia } \\
\hline $\begin{array}{l}\text { 13. ¿Ha sentido que la vida en general ha } \\
\text { sido menos agradable? }\end{array}$ & 212 & 53 & 133 & 33 & 46 & 11.41 & 8 & 2.0 & 4 & 0.99 & 403 & 100 \\
\hline $\begin{array}{l}\text { 14. ¿Ha sido totalmente incapaz de } \\
\text { realizar sus actividades diarias? }\end{array}$ & 264 & 65.5 & 115 & 28.5 & 17.0 & 4.2 & 5 & 1.2 & 2 & 0.5 & 403 & 100 \\
\hline
\end{tabular}




\section{DISCUSIÓN}

En esta investigación sobre la calidad de vida relacionada con salud bucal en una muestra de conveniencia en adultos en la Ciudad de Quito-Ecuador se pudo observar que en cuanto a los aspectos sociodemográficos que caracterizaron la muestra; hubo una ligera predominancia del sexo femenino sobre el masculino, una alta presencia de los grupos de edades comprendidos de 18 a 44 años y un muy elevado porcentaje de individuos con ingresos inferiores a los $\$ 400$ mensuales.

Respecto a la utilización de los servicios de atención odontológica se reportó una alta proporción de individuos que utilizaban los servicios privados o particulares y también se encontró un gran porcentaje de individuos que no poseían seguro de atención dental.

En relación a la CVRSB se pudo observar que la dimensión malestar psicológico reportó un porcentaje significativo de individuos señalaron que a menudo les preocupaba los problemas con su boca y también se sentían estresados a causa de esos problemas. Esto coincide con estudios previos $^{8,17}$ donde se ha evidenciado que las dimensiones psicológicas han sido reportadas como las más afectadas.

Los niveles de ingresos y los servicios de atención de la salud bucal son variables importantes que inciden en la calidad de vida y de salud de la población en general y en particular de la salud bucal. En esta investigación un muy elevado porcentaje de los individuos encuestados auto informó un ingreso mensual inferior al salario básico unificado (SBU) establecido en Ecuador, el cual es en la actualidad de USD 400 mensuales. ${ }^{20}$ y no poseer de seguro dental.

Chafeer et $\mathrm{al}^{6}$ sostienen que las familias que enfrentan mayores desventajas sociales, pueden auto informar un menor impacto en la CVRSB y señala que los individuos cuando enfrentan necesidades en competencia, pueden restar importancia al impacto de las afecciones bucales en relación con otros factores estresantes o prioridades que se consideren más inmediatas.

Así mismo, Ling Sun $^{21}$ reportó que el ingreso familiar fue el que tuvo el mayor impacto en la CVRSB en los adultos jóvenes de su estudio.

Sergei et $\mathrm{al}^{22}$ plantean que alguien con baja salud bucal y expectativas bajas puede no considerar que tiene una disminución de su CVRSB y en consecuencia reportar estar satisfecho. Por el contrario, individuos que poseen una buena salud oral y altas expectativas pueden percibir una baja CVRSB, incluso debido a problemas bucales menores, e informar estar insatisfechos.

La percepción de la CVRSB auto-informada depende de las expectativas y experiencias del individuo, que variaran según factores psicológicos, socioeconómicos, demográficos y culturales. $\mathrm{La} \mathrm{FDI}^{23}$ denomina a estos factores como moderadores ya que son elementos que determinan o afectan la forma en que una persona califica su salud bucal e incluyen la edad, la cultura, los ingresos, y las expectativas entre otros.

Respecto a los elementos culturales estos están relacionados con las creencias, actitudes y percepciones. Las nociones y prácticas sobre la salud y la enfermedad que manejan los diversos grupos humanos, propias de cada pueblo, representan un conjunto de ideas y creencias que son aprendidas en las distintas etapas de la socialización e insertas dentro de una visión del mundo, tienen lugar dentro de un sistema lógico cargado de simbolismo, tradiciones y valores que a su vez modelan sus comportamientos e interpretaciones ${ }^{24}$. De allí que las creencias ideas y preconceptos relacionadas con la salud/enfermedad, pueden también ejercer influencia en la percepción de los individuos respecto a la CVRSB.

Las medidas subjetivas de la calidad de vida pueden reflejar expectativas diferentes dependiendo del contexto sociocultural donde se desenvuelven los individuos lo cual puede tener implicaciones para la utilización de los servicios, la evaluación de las intervenciones de salud bucal, la cuantificación de la morbilidad de la enfermedad en los grupos de bajo nivel socioeconómico ${ }^{6}$ y la autopercepción de la salud bucal. 
Existe un reconocimiento creciente de que la salud oral tiene un importante impacto en la vida social y psicológica de los individuos por lo que cada vez más están siendo tomados en cuenta y utilizadas para las evaluaciones en la vigilancia de la salud bucal. Sin embargo, las medidas de autopercepción de la CVRSB, no deben ser consideradas como única forma de evaluación, sino que siempre deben ser integradas a las evaluaciones clínicas, a fin de evitar la subestimación de las necesidades de salud bucodental sobre todo en las comunidades más desfavorecidas ${ }^{6 .}$

Estas consideraciones devienen de la argumentación de que las poblaciones en desventaja social pudieran darles mayor prioridad a otras necesidades más perentorias y en consecuencia subvalorar su CVRSB. De allí que se requiere de un enfoque exhaustivo e integral que aportaría informaciones y evidencias confiables a los organismos del Estado respecto al diseño, ejecución, evaluación y financiamiento de políticas y estrategias para el componente bucal de salud por ciclos de vida en cada uno los sectores poblacionales.

La calidad de vida relacionada con la salud oral tiene implicaciones importantes para la práctica clínica y de investigación odontológica, ya que centran sus enfoques en cómo los pacientes perciben su salud bucal y necesidades de tratamiento y la eficacia del mismo ${ }^{25}$.

\section{CONCLUSIÓN}

Se concluye que, respecto a la CVRSB, solo en la dimensión malestar psicológico un porcentaje importante de los individuos encuestados auto-informaron una percepción negativa. No así en las demás dimensiones. Los resultados de las mediciones de las múltiples dimensiones del OHIP-14 pudieron estar influenciados por el nivel de ingreso de los individuos lo que a su vez los ubica como población vulnerable y desfavorecida socialmente y ello pudo intervenir en la percepción de su CVRSB ya que al enfrentar necesidades más urgentes para sus condiciones materiales de vida podrían estar restando importancia al impacto de las afecciones bucales.

Las limitaciones de este estudio estuvieron relacionadas con la aplicación del instrumento de recolección de información el cual fue auto-administrado y enviado a través de las plataformas virtuales y no a través de una entrevista con lo cual pudo presentarse problemas de comprensión de las preguntas formuladas debido a la naturaleza multidimensional de las variables contempladas en el instrumento OHIP-14 situación que pudo haber introducido sesgo en cuanto a la información auto-referida.

Se sugiere la realización de estudios longitudinales utilizando las medidas de determinación de la CVRSB mediante la aplicación del OHIP-14 otras medidas relacionadas con la misma según grupos poblacionales específicos y que incluyan además el examen clínico para determinar las diferentes afecciones bucales que padece la población. Así mismo se sugiere que la encuesta nacional de salud bucodental incluya estas medidas de autopercepción de la CVRSB proporcionando así mejores y mayores niveles de evidencia sobre el perfil de salud bucal de la población ecuatoriana.

\section{REFERENCIAS}

1. Urzúa MA, Caqueo-Urízar A. Calidad de vida: una revisión teórica del concepto. Quality of life: a theoreticalreview. Ter Psicol. 2012; 30 (1): 718-4808.

2. Bowling, A. What things are important in people's lives? A survey of the public's judgments to inform scales of health related quality of life, Social Science and Medicine, 1995; 41:1447-1462

3. Schwartzmann Laura. Calidad de vida relacionada con la salud: aspectos conceptuales. Rev Cienc. enferm. 2003. 9(2): 09-21. 
4. Robles-Espinoza Andrea Isabel, Rubio-Jurado Benjamín, De la Rosa-Galván Edith Vanessa, Nava-Zavala Arnulfo Hernán. Generalidades y conceptos de calidad de vida en relación con los cuidados de salud. El Residente. 2016; 11 (3): 120-125

5. Organización Mundial de la Salud. Study protocol for the World Health Organization project to develop a Quality of Life assessment instrument (WHOQOL). Qual Life Res. 1993; 2 (2): 153-159.

6. Chaffee Benjamin W, Humbert Rodrigues Priscila, Floriani Kramer Paulo, Vítolo Márcia Regina, Feldens Carlos Alberto Oral health-related quality-of-life scores differ by socioeconomic status and caries experience. Community Dent Oral Epidemiol.2017 Jun;45(3):216-224 DOI: 10.1111/cdoe.12279

7. Martins MT, Sardenberg F, Vale MP, Paiva SM, Pordeus A. Dental caries and social factors: impact on quality of life in Brazilian children. Braz Oral res. 2015; 29(1):1-7 disponible en https://www.ncbi.nlm.nih.gov/pubmed/26892354

8. Diaz-Cardena, S.; Meisser-Vidal, M. A.; Tirado-Amador, 1. R.; Fortich-Mesa N.; Tapias-Torrado, L y Gonzalez-martinez, F. D. Impacto de salud oral sobre calidad de vida en adultos jóvenes de clínicas odontológicas Universitarias. Int. J. Odontostomat. 2017.11(1):5-11

9. Spanemberg JC; Cardoso JA; Slob EMGB; López-López J. Calidad de vida relacionada con la salud bucal y su impacto en adultos. J Stomatol Oral Maxillofac Surg. 2019; 120 (3): 234-239 DOI: 10.1016 / j.jormas.2019.02.004

10. Yactayo-Alburquerque MT, Alen-Méndez ML, Azañedo D, Comandé D, Hernández-Vásquez A. Impact of oral diseases on oral health-related quality of life: A systematic review of studies conducted in Latin America and the Caribbean. 2021; 16(6): e0252578 doi: 10.1371/journal.pone.0252578

11. Zambrano O, Fong L, Rivera L, Calatayud E, Hernández J, Maldonado A, Rojas-Sánchez F, Principe S, Pérez G, Navarro T, Acevedo A. Impacto de la caries de infancia temprana en la calidad de vida del niño zuliano y su familia. Odous Científica. 2016; 16(2): 8-17

12. Martins MT, Sardenberg F, Vale MP, Paiva SM, Pordeus A: Dental caries and social factors: impact on quality of life in Brazilian children. Braz Oral res. 2015; 29(1):1-7 disponible en https://www.ncbi.nlm.nih.gov/pubmed/26892354

13. Guedes Renata S., Ardenghi Thiago M., Chaiana Piovesan,Bruno Emmanuelli, Mendes Fausto M. Influence of initial caries lesions on quality of life in preschool children: a 2-year cohort study Community Dentistry and Oral Epidemiology.2016.44(3): 292-300

14. Agudelo-Suárez A, Vásquez-Hernández A, Zapata-Villa C. Calidad de vida relacionada con la saludbucal en mujeres en situación deprostitución en Medellín (Colombia) y sus factores relacionados. Rev.CES Odont 2017; 30(2): 3-15

15. Cerón-Bastidas XA. Calidad de vida y su relación con la salud oral en personas de la tercera edad. Rev Nac Odontol. 2014;10(19):83-9. doi: http://dx.doi.org/10.16925/od.v10i19.853

16. Torres-Vanegas Manuela; Cardona-Arango Doris. Calidad de vida relacionada con la salud bucal en adultos mayores en un programa de salud en Envigado, Colombia, 2018. Rev Fac Odontol Univ. 2020. 32(1)1 Doi: https://doi.org/10.17533/udea.rfo.v32n1a4

17. Padilla-Sánchez ML, Saucedo-Campos G, Ponce-Rosas ER, González-Pedraza A. Estado de dentición y su impacto en la calidad de vida en adultos mayores. Rev. CES Odont 2017; 30(2): 16-22.

18. Haag DG, Peres KG, Balasubramanian M, Brennan DS. J Oral Conditions and Health-Related Quality of Life: A Systematic Review Dent Res. 2017 Jul; 96(8):864-874

19. Cantoni Rabolini Mónica Nélida. Técnicas de muestreo y determinación del tamaño de la muestra en investigación cuantitativa. Revista Argentina de humanidades y Ciencias Sociales.2009; 7(2). Disponible en https://www.sai.com.ar/metodologia/rahycs/rahycs_v7_n2_06.htm

20. Ministerio del trabajo. Ecuador. Disponible en https://www.trabajo.gob.ec/el-salario-basico-unificado-sbupara-el-2021-sera-de-usd-400/

21. Ling Sun, Hai Ming Wong,Colman P. J. McGrath. The factors that influence oral health-related quality of life in young adults Health Qual Life Outcomes. 2018; 16: 187. 
22. Sergei N. Drachev, Tormod Brenn, Tordis A. Trovik. Oral Health-Related Quality of Life in Young Adults: A Survey of Russian Undergraduate Students Int J Environ Res Public Health. 2018; 15(4): 719. doi: $10.3390 /$ ijerph15040719

23. World Dental Federation-FDI. Salud bucal y calidad de vida.2015. Disponible en https://www.fdiworlddental.org/oral-health-and-quality-life

24. Paredes Gustavo. Antropologia de la salud y la enfermedad: Principales enfoques Teóricos Revista Academia, 2014.12(30):87-99

25. Daniela von Kretschmann San Martina, Alejandro Torres Varela,Marcial Sierra Fuentes,Julieta del Pozo Bassic, Rodrigo Quiroga Aravenad y Rodrigo Quiroga del Pozoe. Rendimiento masticatorio y nivel de satisfacción de pacientes tratados con prótesis totales en la Universidad Mayor. Rev Clin Periodoncia Implantol Rehabil Oral. 2015;8(1):17-23.

\section{ANEXO 1}

Aprobación del comité de bioética

\section{8) Universidad \\ COMITÉ DE BIOÉTICA DE LA UNIVERSIDAD CATÓLICA DE CUENCA}

Cuenca, 3 de noviembre de 2020

El Comité de Bioética de la Universidad Católica de Cuenca en uso de sus atribuciones legales y reglamentarias, en especial las relacionadas con la revisión de los aspectos bioéticos de los proyectos de investigación y considerando:

1. Que el Comité de Bioética tiene como funciones principales:

1.1. Proponer para su aprobación al Comité Central de Bioética de la Universidad, las normas que permitan la mejor evaluación del componente bioético de los proyectos de investigación.

1.2. Verificar el respeto de los derechos de los pacientes, a su libre participación, comunicación plena y clara con el investigador.

1.3. Revisar toda la información sobre financiamiento, patrocinadores, afiliaciones institucionales y posibles conflictos de interés e incentivos para las personas participantes.

1.4 Consultar con el Comité Central de Bioética de los proyectos de investigación que involucren experimentaciones complejas desde el punto de vista de su componente bioético.

2. El investigador RONALD ROOSSEVELT RAMOS MONTIEL, solicitó a este comité aval para la ejecución del estudio "CALIDAD DE VIDA RELACIONADO CON LA SALUD BUCAL EN ADULTOS DE LA CIUDAD DE QUITO”.

En sesión del Comité de Bioética del 03 de marzo de 2020, determinó lo siguiente:

El comité considera: que este trabajo guarda coherencia conceptual y metodológica en cada uno de los apartados. Está bien escrito y cumple con los elementos como proyectos de investigación. El Comité de Bioética conceptúa que el riesgo de la revisión es "BAJO RIESGO" y otorga el AVAL al tema: "CALIDAD DE VIDA RELACIONADO CON LA SALUD BUCAL EN ADULTOS DE LA CIUDAD DE QUITO”.

El investigador RONALD ROOSSEVELT RAMOS MONTIEL, tendrá la responsabilidad de informar cualquier modificación al articulo, reportar todo evento adverso, responder solicitamente a los requerimientos del Comité de Bioética evaluador y en general ceñirse estrictamente al marco jurídico que regula la investigación biomédica con seres humanos.
Atentamente;

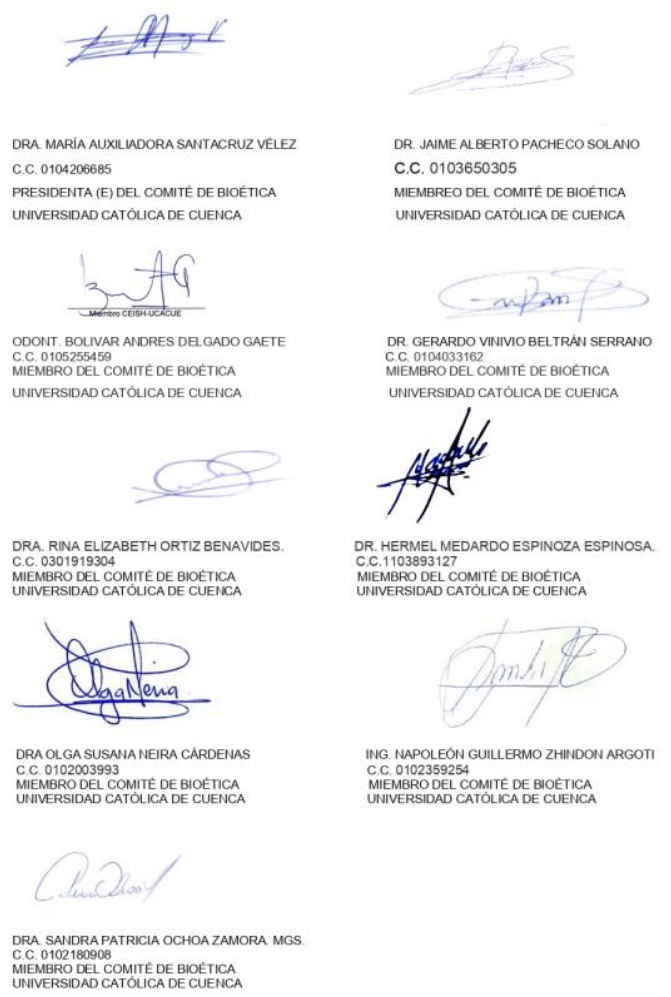
MEEMBRO DEL COMITE DE BIOÉTICA
UHIVERSIDAD CATOLICA DE CUENCA

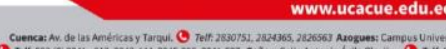

